\title{
Deep 3D Dynamic Object Detection towards Successful and Safe Navigation for Full Autonomous Driving
}

This paper was downloaded from TechRxiv (https://www.techrxiv.org).

LICENSE

CC BY 4.0

SUBMISSION DATE / POSTED DATE

$17-01-2022$ / 24-01-2022

\section{CITATION}

Wijesekara, Patikiri Arachchige Don Shehan Nilmantha (2022): Deep 3D Dynamic Object Detection towards Successful and Safe Navigation for Full Autonomous Driving. TechRxiv. Preprint. https://doi.org/10.36227/techrxiv.18517925.v1

$\mathrm{DOI}$

10.36227/techrxiv.18517925.v1 


\title{
Deep 3D Dynamic Object Detection towards Successful and Safe Navigation for Full Autonomous Driving
}

\author{
Patikiri Arachchige Don Shehan Nilmantha Wijesekara ${ }^{a}$ \\ a Department of Electrical and Information Engineering, Faculty of Engineering, University of Ruhuna, Galle, 80000, Southern Province, Sri \\ Lanka; nilmantha@eie.ruh.ac.lk
}

\begin{abstract}
Infractions other than collisions are also a crucial factor in Autonomous driving since other infractions can result in an Accident. Most of the existing work has been tested for Navigation, collisions and least tested for other infractions such as off-road driving and not obeying road signs. We present an imitation learning based Base Model called CILDO and an optimized model of the base model optimized using an and additional traffic light detection branch and Deep Deterministic Policy Gradient based Reinforcement Learning called CILDOLI-RL. The proposed base

model is designed to detect dynamic objects by developing the vision for semantic features, depth and motion which is crucial in Autonomous driving. The CILDO-RL model presented in this paper achieves highest score for the newly introduced No-OTHERINFRACTION benchmark ensuring safe autonomous driving while the base CILDO model achieves the best performance in Navigation under Urban or rural dense traffic environments. Further, this work makes a comparison on the computational complexities of the driving models.
\end{abstract}

Keywords: Autonomous driving, CILDO, Imitation learning, reinforcement learning, Infractions

\section{Introduction}

\subsection{Background}

\subsection{1. traditional approaches}

Autonomous driving and navigation have been a problem which people had been trying to solve for the past three decades. That is people have been attempting to recognize a pattern in sensory inputs to map into driving functions. The Early solutions had been more biased towards heuristic approaches which decisions for navigation is provided to the robot using algorithms [1]. These systems can be thought of as a modular pipeline consisting mainly of three pipeline stages namely perception, local planner and continuous controller in order. In such systems; the sensor data is provided to a perception module which then plans the motion by generating waypoints or detecting lane edges to follow to decide the next state and finally to provide to a control algorithm to get the desired navigation function for the robot [19]. In [2]; a SONAR based crop row mapping technique is used which is a row following technique along with fuzzy logic control for navigation. Proportional Integral and Derivative (PID) control for the steering of a tractor had been used using geomagnetic sensor readings [3]. Some have used image processing techniques to extract features from cameras to aid in taking navigation decisions. [4] presents such work in an agricultural environment which crop row lines were extracted from raw camera images using morphological image and threshold functions which is still a row following technique. These techniques can be thought of as Edge/line detection techniques to aid taking driving decisions.

Sensor data fusion is another key research area in autonomous navigation systems the fusion process directly affects the outputs of the system which are the actions of the robot. Kalman filter has been extensively used in traditional systems for autonomous navigation due to its robustness to multisensory noise [6]. A recent work shows that particle filter performs better than Kalman filter for navigation in orchards [5]. This classical approach belongs to the use Simultaneous Localization and Mapping (SLAM) [7] using Kalman Filter, graphs, particle filter, monocular visuals.

\subsection{2. modern approaches}

More recent approach is to aid machine learning in autonomous navigation. Many have used machine learning for computer vision which is a sub system in modern autonomous driving. Specifically, traditional modular pipeline is constructed with the help of Convolutional Neural Networks (CNNs) which recognizes patterns in sensor data [8]. Similar to crop row or lane identification using image processing techniques; modern approaches use Recurrent Neural Networks (RNNs) for the same task; but with advancements such as detection of lanes using a stack of images which can overcome partial sensor observations [17]. Some use only CNNs for real-time as semantic segmentation with bounding boxes considering the fast inference [18]. Modular pipeline involves a separate perception module which can be optimized using depth, semantic features and a driving module where perception from computer vision is used in deriving driving functions such as controlling throttle and steering [8].

Another approach is using imitation learning which involves either a trained human or a robot performing tasks which is imitated by a learner to find a policy to map a given state to a set of driving actions [9] by detecting 
patterns in input data which is a supervised learning approach. Even though the concept of application of imitation learning for autonomous driving runs back to late 1990s [12]; it had gained recognition in the recent past due to advancement in Deep learning. Originally, imitation learning for autonomous driving has been trained using the DAGGER (Dataset aggregation) algorithm [9]. An online imitation learning system for off-road autonomous driving has proved to perform better than batch imitation learning systems. In [10]; authors propose an additional execution layer to ensure the safety and feasibility by tracking the short-term trajectories extracted from the policy layer formed by a small neural network trained using imitation learning. However, the said work is limited to vehicle following, lane following and overtaking in straight roads and does not address complex urban driving scenarios. In [11]; the bird's view of the driving environment is used for imitation learning to get the driving policy integrated with a safety controller and a trajectory tracking module to ensure safe driving. Use of RNNs for imitation learning based on crowd sourced video data is presented in [13]. A more recent approach is to use conditional imitation learning which is a blended version of conventional modular approach with imitation learning for high level end-to-end driving at the same time responding to low level intermediate commands (Eg: route commands) as conditions between end-to-end driving during test phases [14]. This work removes the error due to assumption that sensory perception alone contributes to take driving actions in end-to-end driving. This system provides alternative paths to get to the same location which agrees with the practical scenarios. This system instead of end-to-end mapping a given state directly to steering and throttle; maps the state (camera readings) to semantic features, geometry (monocular depth) and motion (monocular optical flow) to be fused in an intermediate layer and provided to the control layer for controlling function [14, 15] having better results than the preceding end-to-end training method.

Reinforcement learning is another one of the major research areas for autonomous driving. Reinforcement learning can be thought of as recognizing a pattern in states to map a given state to an action such that an agent in a given environment performs actions in order to maximize future rewards in future states. Reinforcement learning has been used for lane following in high curvature roads [22]. In [21], depth information of the monocular cues is mapped for steering direction control using reinforcement learning to avoid obstacles. Driver assisting corporative adaptive cruise control system with vehicle-to-vehicle communication for longitudinal control of vehicles using reinforcement learning to optimize the control policy has been used [23]. As a sub problem of autonomous driving; Reinforcement learning has been used for overtaking decision making in highways using Q learning [25]. Another subproblem is automatic lane change that can change lane even under unforeseen instances using reinforcement learning is carried out in [32]. In [26]; it shows how reward functions for deep reinforcement learning can be designed considering collision, keeping in lane, speed, distance to goal etc. In [27]; the authors investigate on how DRL can be used to effectively learn how to pass occluded intersections overcoming heuristic approaches. The camera vision is encoded into a latent state and then a deep Q network agent is trained in [33] to obtain a model free deep RL. In multifidelity reinforcement learning; the policies for low fidelity simulators were transferred to high fidelity simulators for exploration as heuristics to find optimal policies with less data [28]. A constrained Markov Decision Process (MDP) called survival oriented RL which takes survival (Negative avoidance) as the first priority rather than maximizing reward is considered in [29] for ensuring safety. Multi-Agent RL has been used for high level strategic decision making such as overtaking, following vehicles using dynamic coordination graphs [30]. Despite the super-human performance of reinforcement learning in gaming environments; the work in [31] shows the requirement for a deep deterministic policy gradient algorithm to cater complex and continuous state, action space in autonomous driving. A deep RL framework for autonomous driving using sensor fusion and RNN is presented in [34].

\subsubsection{Motivation}

In most of the autonomous driving literature it has been mentioned that a total heuristic system mapping sensor inputs to driving functions is infeasible [9]-[16]. Very significant research findings on autonomous self-driving in urban environments is discussed in [19] which compares three methods of autonomous driving learning policies namely modular pipeline, conditional imitation learning and reinforcement learning. According to the results for urban driving, Imitation learning and modular pipeline approach interchangeably has better performance based on the environment of training and testing. As pointed out in [16] and as proved in [19] imitation learning suffers from data set bias and overfitting giving better performance in environments similar to trained environment and vice versa. Both deep reinforcement learning and imitation learning has proved to be suffering from generalization variability when changing the initialization or training sample order [20, 16, 8].

The paper [20] proves that under dynamic environments; the performance of reinforcement learning degrades. Further, it shows that the hyper-parameter selection and the reward scaling can affect the results which is why the reinforcement learning has outstood both modular approach and imitation learning by a large margin even under new environment for least number of pedestrian collisions in [19] despite its poor driving performance compared to imitation learning and modular approach. According to [16]; imitation learning has poor performance with increased dynamic objects. Collision with a human is the utmost error that can be caused by an autonomous vehicle. Real autonomous vehicles have met with accidents and humans have been injured due to collision where in most cases at the intersections and near the traffic lights [35]. In 2018, a self-driving Uber car killed an innocent pedestrian crossing an intersection [36] in Arizona. Therefore, for the driving policy; the human safety factor should be embedded. The best approach to include in using reinforcement learning according to literature. But, due to its bad driving performance, reinforcement learning alone will not perform the intended driving functions compared to imitation and modular approaches. 
A more recent work is the concept of Conditional Affordance Learning (CAL) which combines imitation learning and modular approach together for autonomous driving in urban environments [37]. In that technique, first a neural network is trained to learn a set of driving environment parameters called affordances and then affordances are trained separately to driving functions. In such techniques, one stage consists of a neural network with perception module for affordances and a controller with conventional PID controller and Stanley Controller for longitudinal and lateral control. In such techniques; the performance depends on the driving controller's accuracy and as proved in results of [16], end-to-end imitation learning models have better navigation performance than CAL. Therefore, our approach deviates from modular pipeline architecture and follows a complete neural network mapping inputs directly to longitudinal and lateral control. Further, recently the concept of Controllable Imitative Reinforcement learning (CIRL) had also proved promising results than each of the 3 individual approaches by combining imitation learning with reinforcement learning [38]. Two models proposed by us are designed inspired by the work of [8], [16], [38]

\subsection{Problem Statement}

The problem is the lack of a driving model for autonomous driving with increased navigation success rate and at the same time maintaining the safety of navigation. To address that problem, driving models are proposed by considering the performance for safety using the novel NoOTHERINFRACTION benchmark proposed in this research.

\subsection{Objectives}

- Inspired by work of [8] and CILRS [16]; we perform the dynamic object detection by joint optimization for semantic feature detection, depth detection and speed prediction which outperform the existing imitation learning driving models.

- Inspired by work of [38], in proposed Actor-Critic model; Infraction minimization is performed in terms of vehicle speed, road sign obeying and driving in correct lane which outperform other autonomous driving models in terms of newly introduced NoOTHERINFRACTION Benchmark introduced in this work.

- Models will be compared to investigate each model's computational complexities.

\section{Methodology}

\subsection{Driving Models}

\subsubsection{Proposed Actor Model (CILDO)}

Actor model is trained and developed using Imitation learning. Imitation learning involves recording a set of Ac- tions $(A)$ for a set of Observations $(O)$, Measurements $(M)$ and Navigation instructions $(I)$. The action space involves 8 actions with 2 selected actions for a given I in Conditional Imitation learning. The two actions contain values for (brake+throttle) and steering. There are 4 Navigation instructions namely "Follow Lane", "Turn Right at Junction", "Turn Left at Junction", "Go Straight at Junction". These are instructions that should be given by a Navigation planner such as Google Maps [47] to decide where to when the vehicle approaches and exit a junction. Navigation planning is independent of autonomous driving yet; autonomous driving relies on navigation planning. Observation set consists of a single RGB camera image during predictions and it will additionally contain Semantic camera images and depth camera images during model fitting. The measurement obtained is speed. The model while training will understand 3 dimensional dynamic objects to map an algorithm for driving. Therefore, we name this method as Controllable Imitation Learning Dynamic Objects (CILDO). The conditional imitation learning can be expressed using the Equation 1

$$
A_{t}=\theta\left(O_{t}, I_{t}, M_{t}\right)
$$

As seen in Equation 1; it is a problem of finding the optimal policy $(\theta)$ to map inputs into outputs where subscript $\mathrm{t}$ stands for the sample of the corresponding set at $t^{\text {th }}$ time step.

Recently developed MobileNetV2 is used as the backbone image feature recognition network due to its higher generalization capability, higher convergence and higher accuracy $[43,45]$ over other image recognition models. Inspired by evaluation of vision based driving models [41], we use speed, depth, dynamic vision, segmentation to optimize backbone network which forces the backbone network to train for identifying 3 dimensional dynamic $o b$ jects which are used as perception when determining driving tasks. We use dropout of 0.2 in convolutional layers to prevent the neural networks from overfitting and not generalizing $[42,16]$. That is because we want the Neural network to generalize to unseen environments (New Town, new weather) as a regularization step.

We use a modified version of U-Net [44] for image encoding and decoding for both image segmentation and depth mapping. Specifically, the encoder is implemented using MobileNetV2 [45] as a pre-trained encoder from Keras applications and decoder mainly derived using pix2pix [46] by up sampling the encoder output which implementation varies depending on the task which will ultimately lead to one encoder and 2 decoders. To embed dynamic vision in the encoder; we predict speed of the vehicle using RGB image. Part of the expanded model is given in Figure 1. 


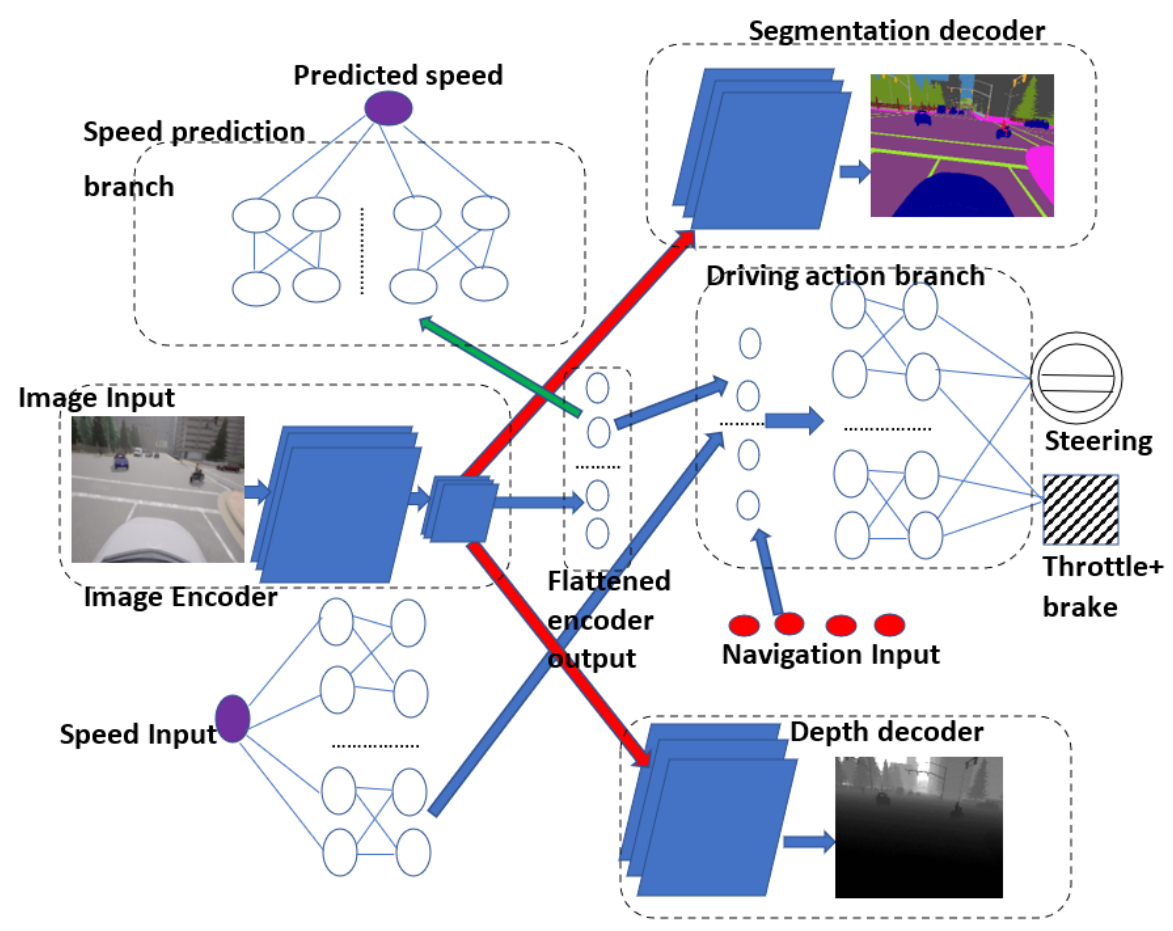

Figure 1: CILDO Model

As evident from Figure 1, The output of the encoder is concatenated with expanded dense layer from the speed measurement to obtain the perception for driving. That is because the encoder is optimized for 3D static object detection by 2 decoders implemented for semantic feature detection, depth vision and at the same time optimized for speed related features from speed prediction branch. Hence, all 3 types of perception required for autonomous driving namely semantic features, depth and dynamic vision will be completed. Therefore, the loss function (L) for the model will be composed of 4 terms as shown in Equation 2

$$
\begin{aligned}
\operatorname{Loss} & =\left(\sum_{i, j}^{W, H} \hat{P}_{s(i, j)}-P_{s(i, j)}\right)^{2}+\left(\sum_{i, j}^{W, H} \hat{P}_{d(i, j)}\right. \\
& \left.-P_{d(i, j)}\right)^{2}+\left(\hat{M}_{t}-M_{t}\right)^{2}+\left(\hat{A}_{t}-A_{t}\right)^{2}
\end{aligned}
$$

where $\hat{P}_{s(i, j)}$ is the $(i, j)^{\text {th }}$ pixel value of the predicted segmentation image, $\hat{P}_{d(i, j)}$ is the $(i, j)^{\text {th }}$ pixel value of the predicted depth image, $\hat{M}_{t}$ is the speed prediction and $\hat{A}_{t}$ is the predicted action set.

Figure 2 shows the input image, ground truth segmented image from semantic camera, ground truth depth image from depth camera compared with proposed model's predicted segmented and depth images. The semantic segmentation camera converts a given pixel into one of 13 classes with corresponding color code as given in Table 1 [51].

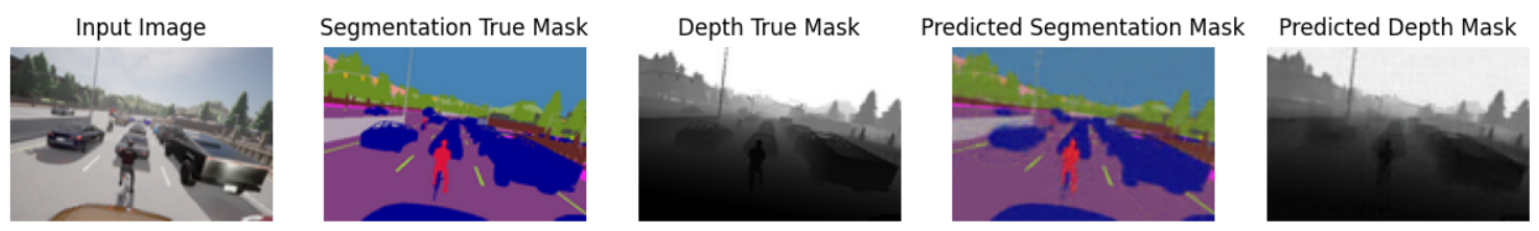

Figure 2: Comparison of Ground truth images and images predicted from the model 


\begin{tabular}{|l|l|l|}
\hline Class & RGB Pixel Value & Color \\
\hline Building & $(70,70,70)$ & \\
\hline Other & $(110,190,160)$ & \\
\hline Vegetation & $(107,142,35)$ & \\
\hline Poles & $(153,153,153)$ & \\
\hline Traffic Lights & $(250,170,30)$ & \\
\hline None & $(70,130,180)$ & \\
\hline Road & $(128,64,128)$ & \\
\hline Road Lines & $(157,234,50)$ & \\
\hline Vehicles & $(0,0,142)$ & \\
\hline Sidewalks & $(244,35,232)$ & \\
\hline Fences & $(150,100,100)$ & \\
\hline Walls & $(145,170,100)$ & \\
\hline Padestrian & $(220,20,60)$ & \\
\hline
\end{tabular}

As observed from Figure 2; the model has been fairly able to predict the semantic and depth features simultaneously.

\subsubsection{Proposed Actor-Critic Model (CILDOLI-RL)}

When compared to the CILDO model, CILDOLI-RL model has an additional loss functions corresponding to detection of traffic light state as shown in Figure 3. We model the detection of 3-way traffic lights at a junction using the neural network to predict from the input image.

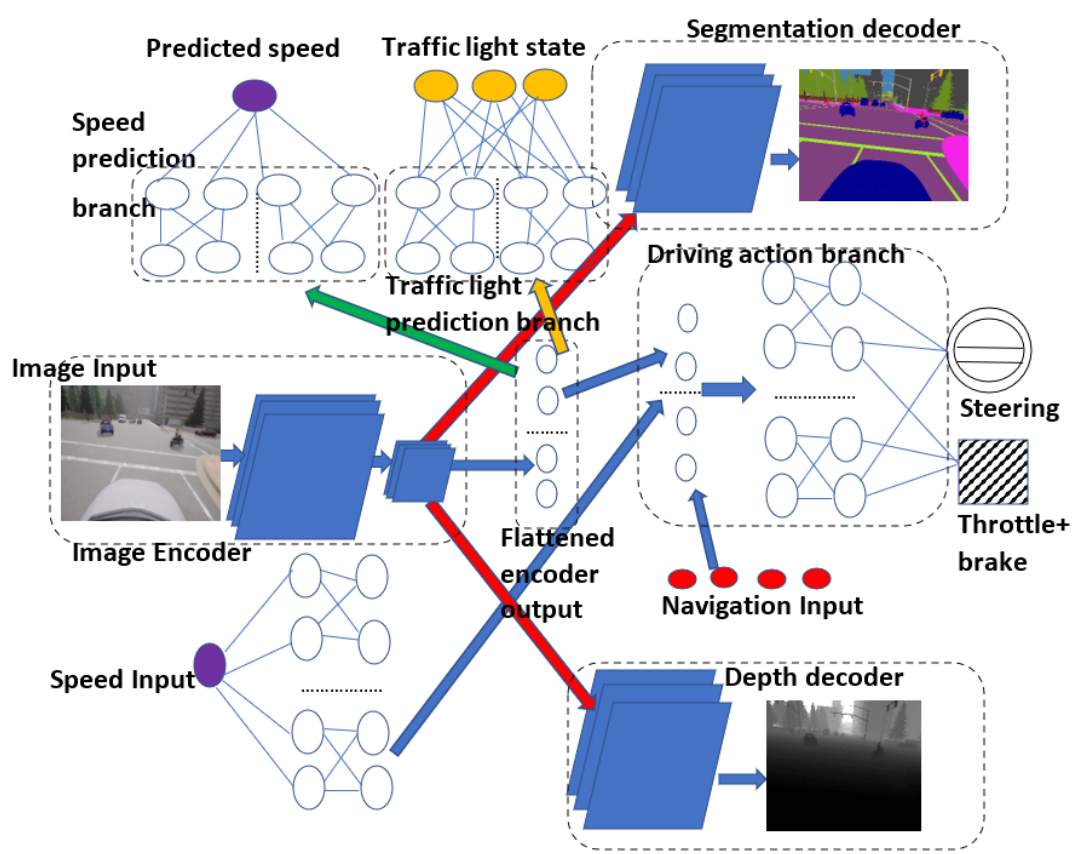

Figure 3: CILDOLI actor Model 
The additional branch corresponds to an additional loss function. So, the loss function for CILDOLI-RL model will be as given in Equation 3.

$$
\begin{array}{r}
\text { Loss }=\left(\sum_{i, j}^{W, H} \hat{P}_{s(i, j)}-P_{s(i, j)}\right)^{2}+\left(\sum_{i, j}^{W, H} \hat{P}_{d(i, j)}-P_{d(i, j)}\right)^{2} \\
+\left(\hat{M}_{t}-M_{t}\right)^{2}+\left(\hat{A_{t}}-A_{t}\right)^{2}+\left(\hat{T_{F t}}-T_{F t}\right)^{2}+\left(\hat{T_{L t}}-T_{L t}\right)^{2} \\
+\left(\hat{T_{R t}}-T_{R t}\right)^{2}
\end{array}
$$

where $T_{F t}, T_{L t}$ and $T_{R t}$ are the ground truth values of the Forward lane Traffic light state, Left Lane traffic light state and Right side lane traffic light state respectively.

Reinforcement Learning. Reinforcement learning allows an Agent to learn how to behave in an environment. For the offline RL system, the environment is the simulated driving environment of CARLA and the Agent is the ego vehicle. However, due to the continuous nature of driving actions and states; initial exploration as in the case of conventional reinforcement learning can result in longer training times and waste of resources. That initial exploration is drastically reduced by learning a base driving policy using imitation learning. A Markov Decision Process (MDP) in Reinforcement learning consists of environmental States $\left(S=s_{1}, \ldots, s_{N}\right)$, Actions $\left(A=a_{1},,, a_{N}\right)$, Transition function which is a probability distribution between states $T\left(s, a, s^{\prime}\right)$ and a Reward function for performing an action at a particular state to transit into a new state $R\left(s, a, s^{\prime}\right)$. The driving process can be considered as a MDP since an action taken does not depend on previous observations and previous actions taken; but only depends on the present observation [48]. The tuple (S, A, T, R) in MDP is called experience and it should be stored in order to take decisions. The collection of such tuples stored over time is called an experience replay. The state and action spaces are not discrete; but continuous variables in autonomous driving. The state $\mathrm{S}$ consists of an Image (I), the speed measurement $(\mathrm{M})$ and Control command $(\mathrm{C})$ for autonomous driving using CILDOLI-RL model.

Since autonomous driving involves continuous domain state-action pairs; recently developed Deep Deterministic Policy Gradient (DDPG) algorithm is used to update the actor and critic's model weights in Reinforcement learning [50].

Deep Deterministic Policy Gradient (DDPG) Method. A deterministic Policy $(\pi: S \mid A)$ is a function belonging to the agent mapping each state (S) input into an action (A). Therefore, the neural network which maps a given state to an action (CILDO) model is a Deep Deterministic Policy (DDP) network. The goal of reinforcement learning is to learn a policy called the optimal policy which maximizes the rewards in long-term basis. In DDPG method, both the actor network policy and the critic network policy should be updated. Both actor and critic are having 2 networks per each called the target model and the training model. Based
For proposed Actor-Critic in particular, for reinforcement learning, we deviate from the batch reinforcement learning with experience replay [24] by only having a replay memory size of 2 only storing the present experience and previous experience.

$Q$-Learning. A state-action value function $Q(s, a)$ calculates how good is to take an action $\mathrm{A}$ in a given state (S) by following a policy $\pi$. It is defined as the expected return from future rewards as shown in Equation 4 [49].

$$
Q^{\pi}(s, a)=E_{\pi}\left(\sum_{k=0}^{\infty} \gamma^{k} r_{t+k} \mid s_{t}=s, a_{t}=a\right)
$$

where $\gamma$ is called the discount rate which is a positive constant less than 1 .

The $\mathrm{Q}$ values need to be updated as rewards are obtained according to the Bellman Equation [48] as shown in Equation 5

$Q_{t+1}\left(s_{t}, a_{t}\right)=Q_{t}\left(s_{t}, a_{t}\right)+\alpha\left(r_{t}+\gamma \max Q_{t}\left(s_{t+1}, a_{t}\right)-Q_{t}\left(s_{t}, a_{t}\right)\right)$

In equation 5, $\alpha$ is the learning rate for updating $\mathrm{Q}$ values which we set to 1 in this research to yield the following simplified equation as shown in Equation 6.

$$
Q_{t+1}\left(s_{t}, a_{t}\right)=\left(r_{t}+\gamma \max Q_{t}\left(s_{t+1}, a_{t+1}\right)\right.
$$

Here, the actor is the autonomous driving policy network $\pi_{a}$. The critic network is a value function which is also a policy neural network $\left(\pi_{c}\right)$ having input as $(\mathrm{S}, \mathrm{A})$ and output as Q. Here, S and A are state and action of the actor network respectively and Q is the corresponding stateaction value. The layer model of the proposed critic network is as shown in Figure 4.

on reinforcement learning using the rewards obtained per actions; the training models of both the actor and critic are immediately updated; but the target models are updated at a selected learning rate $(\tau)$ by using weights from training models to converge target models to training models in the long run. The replay buffer consists of the present experience tuple and the previous experience tuple of the MDP. The DDPG process is as follows. Note that here previous and current experience denotes two consecutive time steps $(t$ and $t+1)$ experience. Following set of actions are repeated for each new state-action pair.

- Reward $r_{t}$ for the previous predicted action is calculated using the reward function.

- For a given state $\left(s_{t+1}\right)$; the target actor model predict an action $\left(a_{t+1}\right)$ using the target actor model's policy $\left(\pi_{a T a r}\right)$ 


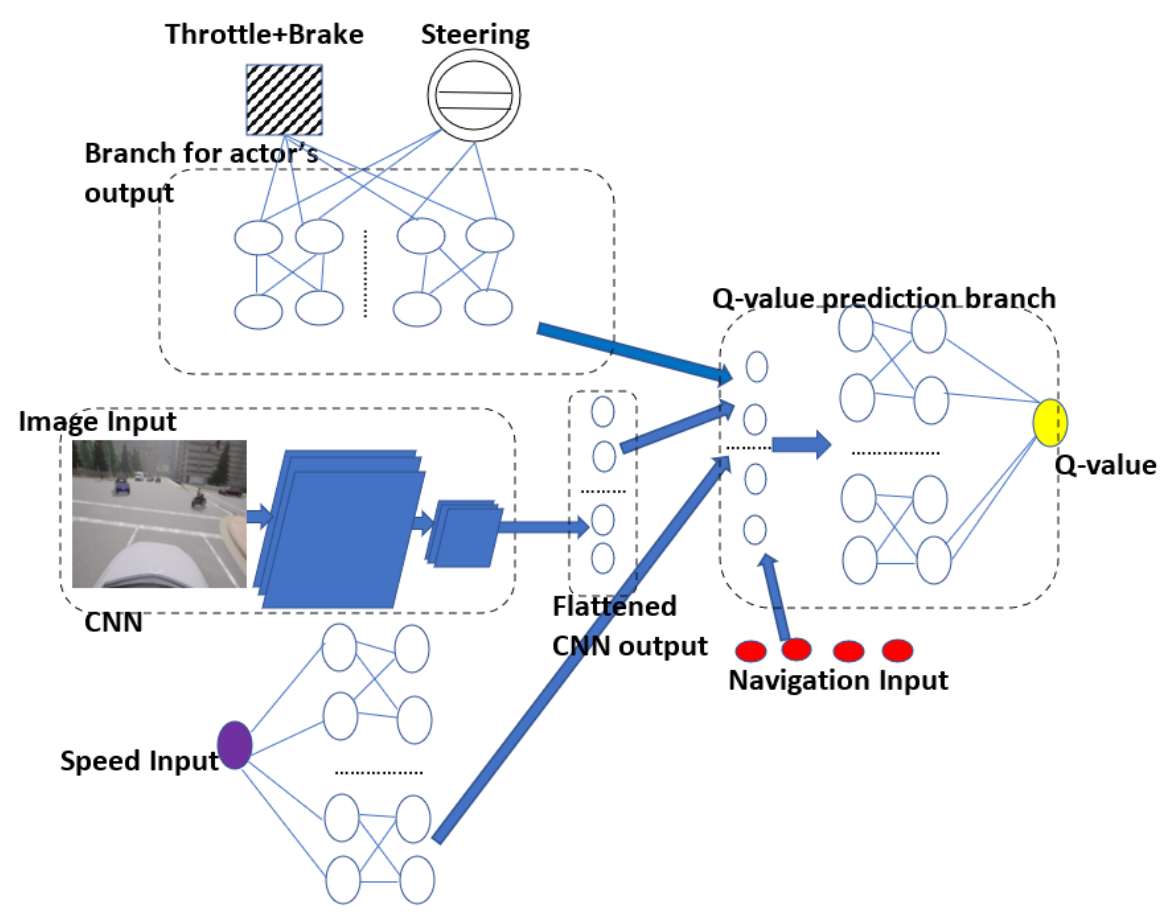

Figure 4: CILDOLI critic Model

- Using the predicted action $\left(a_{t+1}\right)$ and $\left(s_{t+1}\right)$; the target critic model predicts the $Q_{t}\left(s_{t+1}, a_{t+1}\right)$ value for the state-action pair using the target critic model's policy $\pi_{c T a r}$.

- Using the predicted action's reward $\left(r_{t}\right)$ and discount factor $(\gamma)$ and predicted $Q_{t}\left(s_{t+1}, a_{t+1}\right)$ value from previous step; using equation 6; updated $\mathrm{Q}$ value $\left(Q_{t+1}\left(s_{t}, a_{t}\right)\right)$ also called as the one step return is found.

- Now, from the replay buffer, previous state $\left(s_{t}\right)$ is selected. Using $s_{t}, a_{t}$ and training critic model's policy $\left(\pi_{c T r a}\right) ;\left(Q_{t}\left(s_{t}, a_{t}\right)\right)$ is found.

- $\left(Q_{t+1}\left(s_{t}, a_{t}\right)\right)-\left(Q_{t}\left(s_{t}, a_{t}\right)\right)$ is set up as the training critic model's loss.

- The gradient of all trainable weights of the training critic network (DDP) with respect to the critic model's loss computed in previous step is calculated and then weights are adjusted such that the rritic $_{l}$ oss is minimum.

- The gradient of all trainable weights of the actor network (DDP) with respect to the Q value $\left(Q_{t}\left(s_{t}, a_{t}\right)\right)$ predicted by the training critic network called "critic value" is calculated and then weights are adjusted such that the "critic value" is maximized.

- Finally, target actor model and target critic models are updated at a learning rate of $\tau$

Reward Function. We closely associate our reward function as of work in [38]. However there are new components in our reward function and changes to already existing components in [38]. We define the reward function as given in Equation 7 $r=r_{\text {steer }}+r_{\text {speed }} * r_{\text {traffic-light }}+r_{\text {damage }}+r_{\text {off-road }}$

In safe autonomous driving, the vehicle should obey the traffic lights. Therefore, we have introduced a reward for moving in a traffic light as in Equation a.

$r_{\text {traffic-light }}= \begin{cases}-1 & ; \text { moving in red light } \\ +1 & ; \text { moving in green light or unkown light }\end{cases}$

The reward component for speed in [38] does not take into account the speed limits for the lane following function and provides a positive constant reward even at very high speeds. For the vehicle to follow speed limits of the environment, we set the $r_{\text {speed }}$ as in Equation a.

$r_{\text {speed }}= \begin{cases}-10 & \text { if } v>\text { speed limit for follow lane } \\ v & \text { if } v \leq \text { speed limit for follow lane } \\ 40-v & \text {;if } v>20 \text { for straight, left, right } \\ v & \text {;if } v \leq 20 \text { for straight, left, right }\end{cases}$

Here, the speed limit is a variable whose value depends on the road which is driving.

Negative rewards are provided for steering $\left(r_{\text {steer }}\right)$ in opposite directions for turn left or turn right or steering in either direction for going straight. $r_{d}$ amage is composed of negative rewards for collision with a vehicle or pedestrian or any other object. $r_{o f f-\text { road }}$ is composed of negative rewards for either moving in opposite lane or crossing the sidewalk. 


\section{Results}

CARLA is an open-source urban driving simulator used to develop, train and validate autonomous urban driving [19]. We use CARLA version 0.9.12 in our simulations. CARLA runs as a client-server system which can be implemented in Synchronous mode or asynchronous mode. We run all simulations in synchronous mode at a fixed frame rate of 8 FPS where the server waits until a clock tick is heard from the client which removes any possible error due to processing delays such as prediction delays from neural networks.

\subsection{Training conditions}

Since the camera pose being fixed in space can lead to control errors and unexpected behaviors reinforcing each other [37, 39], we randomize the camera position in along 0.10 to 0.25 times the length along positive $\mathrm{x}$ (length - forward direction) direction, 1.5 to 2.0 times the height of vehicle in $\mathrm{z}$ (height) direction from origin, pitch by 270 to 290 degrees, roll from 175 to 185 degrees, yaw from -5 to +5 degrees. Here, the xyz coordinate axes is a non-inertial frame of reference with origin at centroid of the vehicle. In a real-world scenario, this will represent different field of view of different drivers with different height and seat adjustments which is more practical than a fixed first-person camera at front of the ego vehicle which always cannot see its parent vehicle [16]. Town 5 is selected as the training city which is an urban town having multiple cross junctions and a bridge with multiple lanes per direction [40] which is shown in Figure ??. We set the weather randomly as dry or cloudy and change the lighting conditions randomly between Noon,Sunset,Night. We generate regular and Dense traffic conditions as follows for both the training and test conditions. Regular condition is 15 vehicles and 5 pedestrians per town whereas the dense condition is 45 vehicles and 15 pedestrians per town. Training takes place for all empty, regular and urban traffic. In empty conditions there are no pedestrians or other vehicles present in the town except for the ego vehicle. We train each model for any number of epochs until the predicted actions loss is not decreasing. Neural networks are implemented using Tensorflow Version 2.6 and trained using CPU version. The optimizer is selected as Adam with an initial learning rate of 0.0002 which decays by $9 \%$ at the end of each epoch. The loss function is the Mean Squared Error between ground truth values and the predicted values. Figure 5 shows the training progress of each of the driving models which are trained in this context.

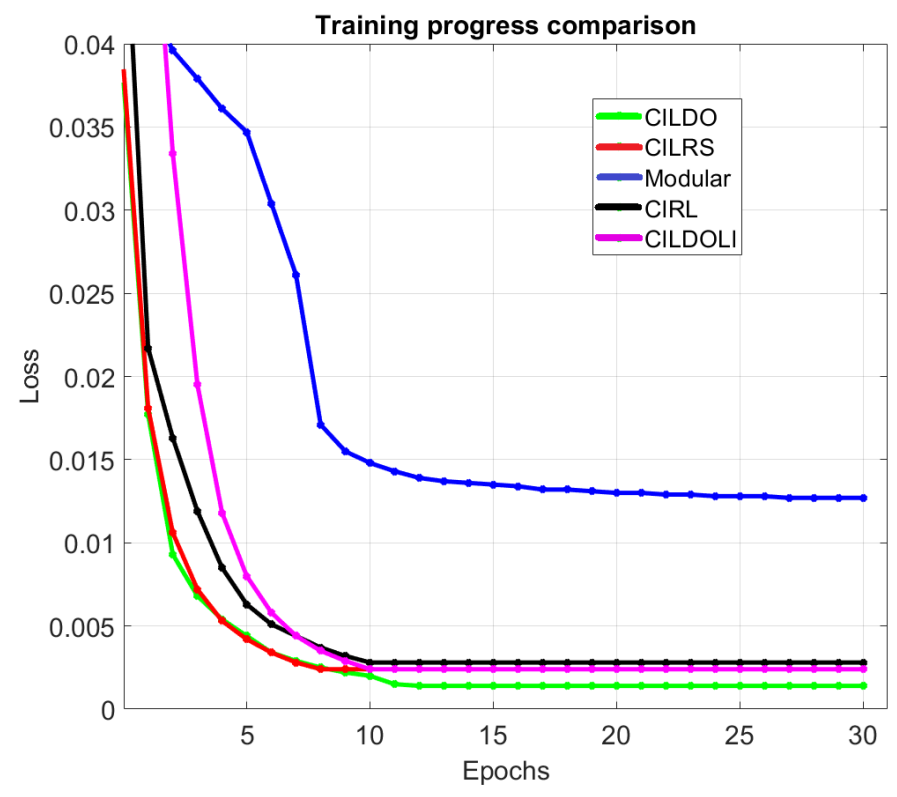

Figure 5: Comparison of Predicted actions Loss vs Epoch during training for Imitation Learning

As evident from Figure 5; the modular approach is having the highest loss out of the methods considered. That is due to the fact that in modular approach; the encoder is pre-trained separately before training the driving model. When the driving model is trained; the encoder's training is frozen so that its weights cannot be adjusted to minimize the predicted actions loss. On the other hand; the proposed CILDO method yields the least loss during training; therefore, higher driving performance can be expected from the CILDO method at the end of training for imitation learning.
The model at the end of each epoch is saved and the model which had least loss is selected for testing. since it has been found in [16]; that too much offline training on the data set can cause dataset bias in imitation learning; we train all models compared in this work for a total of 8.9hour long demonstrations driven by an expert human driver for a fixed frame rate of 8 FPS resulting a total of 256, 890 frames. Here, the navigation planning is also performed by the human driver and those navigation instructions are also recorded. Due to the bottleneck in system memory dur- 
ing training; we resize the collected dataset to image frame resolution fixed at $120 * 80$. For this data set human driver drives the vehicle following all road signs, traffic lights and road rules unlike end-to-end driving as done in [14]. The driving models can be tested for infractions such as not following traffic lights, invading opposite lane or sidewalk also by training for such a data other than benchmarking for navigation and not crashing only. Therefore, we introduce a new benchmark for autonomous driving known as No-OTHERINFRACTION benchmark to further optimize driving and to compare driving models. When we spawn the ego vehicle; we select a random vehicle out of Vehicle blue print library in CARLA from the 4-wheel category except for the class "Fire Truck" which deviates by a large margin from the other vehicle categories in size and driver field of view. In particular we pick a random vehicle of a random brand out of a random class: Car, Van, truck, SUV. For the reinforcement learning; we set the discount rate as $\gamma=0.99$, learning rate in bellman equation $\alpha=1.0$, target model update learning rate $(\tau=0.005)$, the critic's learning rate of 0.001 and actor's learning rate of 0.00001 .

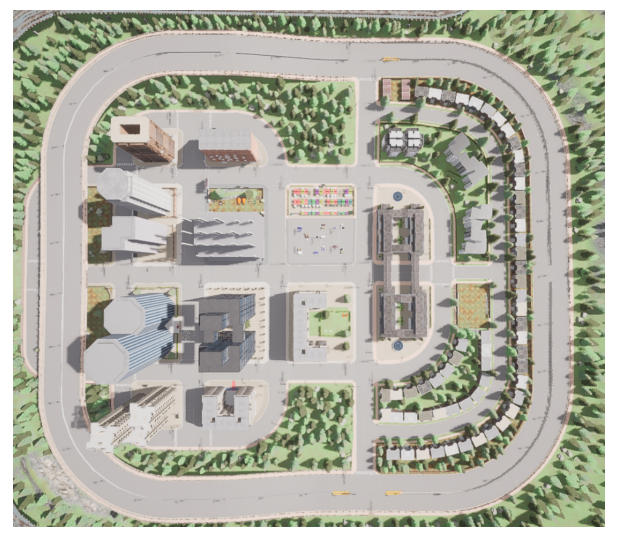

(a) Bird's eye view of Town 05

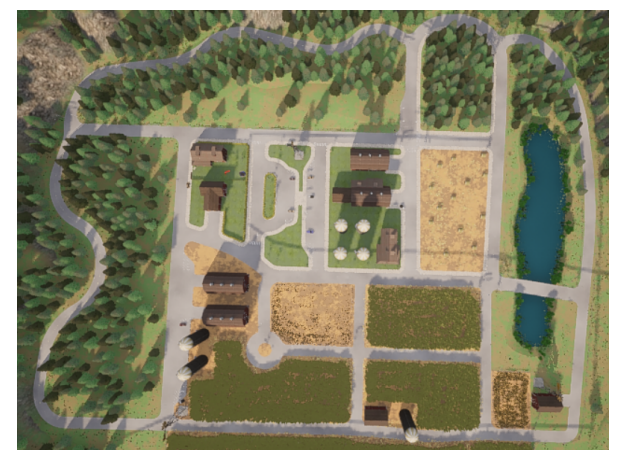

(b) Bird's eye view of Town 07

Figure 6: Training and testing towns

\subsection{Testing conditions}

We fix the camera position at training conditions at 0 distance in $\mathrm{x}$ direction, 0.15 times height in $\mathrm{z}$ direction and pitch at 70 degrees to get the best field of view. An entirely different rural town which is Town 7 is selected as new town which is having only narrow roads and hardly any traffic light [40]. Compared to town 7; town 5 has more $\mathrm{y}$ junctions, $\mathrm{T}$ junctions and turns need more steering with higher accuracy at a junction since the lanes are narrow. So, driving is challenging in town 5. It should be noted that testing takes place both in Town 5 and Town 7. At instances for checking new weather; we set the weather randomly as wet or rainy with random changes in lighting as it was done for the training town: Town 5. Even though the training data set contains both day and night driving to provide data for feature detection under different lighting conditions; we test both in town 5 and town 7 only in day light to have consistency in results. However, we keep random selection of a driving vehicle as it was in training to represent a generalized driving model. Tensorflow CUDA enabled GPU version is used to obtain predictions at a frame rate of 8 FPS. Out of all the possible spawn points for the vehicle from the Town, we select a subset of spawn points by removing bad spawn points such as slopes, junctions, car parks etc. The ego vehicle is spawned at a random spawn point from the filtered sub set in the corresponding town and the vehicle should complete the intended navigation within 5 minutes to successfully complete an episode. The episode does not end at a collision; but all the collisions and other infractions (Not following traffic lights, driving in opposite lane, sidewalk) are recorded to find NoCRASH benchmark and new NoOTHERINFRACTION benchmark introduced in this research.

\subsection{CARLA Benchmark}

The CARLA benchmark [19] benchmarks driving from simple going straight at junction to Navigation (Navigate with left and right turns at a junction) under dynamic objects in the training town and in a new town with a new weather. We select training and test environments as it was specified in section 3.1 and 3.2 respectively. 


\begin{tabular}{|l|l|l|l|l|l|l|l|l|l|l|}
\hline \multicolumn{9}{|c|}{ Training conditions (Dry/Cloudy Urban Town) } & \multicolumn{5}{|c|}{ Testing conditions (Rainy Rural Town) } \\
\hline Task & $\begin{array}{l}\text { CILRS } \\
{[\mathbf{1 6}]}\end{array}$ & $\begin{array}{l}\text { Modular CIRL } \\
{[\mathbf{8}]}\end{array}$ & $\begin{array}{l}\text { Proposed } \\
\text { CILDO }\end{array}$ & $\begin{array}{l}\text { Proposed } \\
\text { CILDOLI- } \\
\text { RL }\end{array}$ & $\begin{array}{l}\text { CILRS } \\
{[\mathbf{1 6}]}\end{array}$ & $\begin{array}{l}\text { Modular CIRL } \\
{[\mathbf{8}]}\end{array}$ & $\begin{array}{l}\text { Proposed } \\
\text { [IL] }\end{array}$ & $\begin{array}{l}\text { Proposed } \\
\text { CILDOLI- } \\
\text { RL }\end{array}$ \\
\hline Straight & 91 & 60 & 92 & $\mathbf{9 4}$ & 86 & $\mathbf{9 4}$ & 70 & 90 & 84 & 80 \\
\hline $\begin{array}{l}\text { One- } \\
\text { Turn }\end{array}$ & 70 & 54 & 76 & $\mathbf{8 0}$ & 64 & $\mathbf{5 7}$ & 11 & 44 & 45 & 40 \\
\hline Navigatiol60 & 36 & 55 & $\mathbf{7 0}$ & 60 & $\mathbf{3 0}$ & 8 & 25 & 25 & 20 \\
\hline Dynamiq & 51 & 14 & 49 & $\mathbf{6 2}$ & 46 & 15 & 4 & 13 & $\mathbf{2 1}$ & 11 \\
\hline
\end{tabular}

When looking at the results in Table 2; it is crystal clear that the proposed CILDO method outperforms all other methods for urban navigation. Next highest performance for the training town is by CILRS followed by proposed CILDOLI-RL. The proposed CILDOLI-RL method had sacrificed part of the navigation performance in order to obtain low other infractions as it will be evident from the following sections. On the other hand, for the rural town with a rainy weather CILRS has outperformed all other driving models for navigation tasks in the empty town (straight, one turn, navigation). The proposed CILDO method has outperformed other driving models again for the Dynamic town navigation in the rural town. This can be explained by the fact that the testing town being a rural town which the semantic segmentation for the proposed methods has less classes to classify under no traffic conditions. But when the rural town is concentrated with pedestrians and vehicles; the conditions become close to Urban with higher classes for semantic segmentation so that Pro- posed CILDO method outperforms other driving models. Therefore, CILDO method has the generalization capability to perform in both urban and rural conditions. The proposed CILDO method is enhanced by semantic feature detection in the Urban town and in rural town under dense traffic conditions thus outperforming the state-of-the art driving models. Further, it is clear that the reinforcement learning has not enhanced navigation performance since CARLA benchmark for CIRL or proposed CILDOLI-RL had not outperformed other models which are entirely modelled from imitation learning.

\subsection{NoCRASH Benchmark}

NoCRASH benchmark has been introduced in [16]; which considers an episode being successful if there is no collision with another vehicle or pedestrian or any other object. Table 3 summarizes the percentage of NOCRASH episodes for each of the driving models compared in this context including the proposed 2 models.

Table 3: NoCRASH benchmark Comparison

\begin{tabular}{|l|l|l|l|l|l|l|l|l|l|l|}
\hline \multicolumn{9}{|c|}{ Training conditions (Dry/Cloudy Urban Town) } & \multicolumn{5}{|c|}{ Testing conditions (Rainy Rural Town) } \\
\hline Task & $\begin{array}{l}\text { CILRS } \\
{[\mathbf{1 6}]}\end{array}$ & $\begin{array}{l}\text { Modular CIRL } \\
{[\mathbf{8}]}\end{array}$ & $\begin{array}{l}\text { Proposed } \\
\text { [ILD }\end{array}$ & $\begin{array}{l}\text { Proposed } \\
\text { CILDOLI- } \\
\text { CLL }\end{array}$ & $\begin{array}{l}\text { CILRS } \\
{[\mathbf{1 6}]}\end{array}$ & $\begin{array}{l}\text { Modular CIRL } \\
{[\mathbf{8}]}\end{array}$ & $\begin{array}{l}\text { Proposed } \\
\text { [38] }\end{array}$ & $\begin{array}{l}\text { Proposed } \\
\text { CILDO } \\
\text { RLDLI- }\end{array}$ \\
\hline Empty & 49 & 81 & 56 & 75 & $\mathbf{8 8}$ & 66 & 84 & $\mathbf{8 7}$ & 76 & 79 \\
\hline Regular & 33 & 73 & 43 & 68 & $\mathbf{8 0}$ & 59 & 67 & 58 & 50 & $\mathbf{7 0}$ \\
\hline Dense & 30 & 50 & 40 & 58 & $\mathbf{6 5}$ & 40 & 50 & 42 & 33 & $\mathbf{5 5}$ \\
\hline
\end{tabular}

According to the results in Table 3, DDPG driven proposed CILDOLI-RL model outperforms all other driving models under an urban driving environment. For the rural town for the case of regular and dense traffic; the proposed CILDOLI-RL model has best NoCRASH benchmark performance as evident in Table 3. However, the NoCRASH benchmark for both the proposed methods are comparatively low for the case of empty town in the rural town due to its vision being more biased to the urban environment semantic object detection.

\subsection{NoOTHERINFRACTION Benchmark}

We introduce the NoOTHERINFRACTION benchmark by defining a success event as if the vehicle does not drive off-road and follows all the road signs. For the simulations of this paper, a road sign only refers to traffic lights and stop signs. but in real driving scenario it refers to other road signs such as speed limit signs, warning signs etc. as well. So, if NoOTHERINFRACTION benchmark is successful; it means that the vehicle has not driven in sidewalk or opposite lane, has stopped at all stop signs and obeyed traffic lights. This benchmark is important because presence of a high score means the model has lower potential for accidents. 


\begin{tabular}{|l|l|l|l|l|l|l|l|l|l|l|}
\hline \multicolumn{9}{|c|}{ Training conditions (Dry/Cloudy Urban Town) } & \multicolumn{5}{c|}{ Testing conditions (Rainy Rural Town) } \\
\hline Task & $\begin{array}{l}\text { CILRS } \\
{[\mathbf{1 6}]}\end{array}$ & $\begin{array}{l}\text { Modular CIRL } \\
{[\mathbf{8}]}\end{array}$ & $\begin{array}{l}\text { Proposed } \\
\text { [ILDO }\end{array}$ & $\begin{array}{l}\text { Proposed } \\
\text { CILDOLI } \\
\text { RL }\end{array}$ & $\begin{array}{l}\text { CILRS } \\
{[\mathbf{1 6}]}\end{array}$ & $\begin{array}{l}\text { Modular CIRL } \\
{[\mathbf{8}]}\end{array}$ & $\begin{array}{l}\text { Proposed } \\
\text { [38] }\end{array}$ & $\begin{array}{l}\text { Proposed } \\
\text { CILDO } \\
\text { RLDOLI- }\end{array}$ \\
\hline Empty & 19 & 22 & 13 & 10 & $\mathbf{5 4}$ & 56 & 71 & $\mathbf{8 1}$ & 69 & 80 \\
\hline Regular & 14 & 18 & 14 & 5 & $\mathbf{5 2}$ & 50 & 58 & $\mathbf{7 5}$ & 55 & $\mathbf{7 5}$ \\
\hline Dense & 0 & 10 & 5 & 0 & $\mathbf{4 5}$ & 45 & 50 & 58 & 44 & $\mathbf{6 7}$ \\
\hline
\end{tabular}

It is evident from Table 4 that in urban environment, the proposed CILDOLI-RL model outperforms all other models for the NoOTHERINFRACTION benchmark by a large margin. That performance gap is due to the fact that none of the other models are not trained or optimized to detect and obey traffic light state while the CILDOLI-RL do both detection by the imitation stage and conditioning to obey it by providing a positive reward at the reinforcement learning stage. The performance gap is lower in the rural town which only has a handful of traffic lights and stop signs unlike in town 05. Possibly due to the advantage in vision in the empty town 07 , CIRL has slightly outperformed the proposed CILDOLI-RL. CIRL and proposed CILDOLI-RL model have equivalent performance in regular traffic and CIRL has inferior performance in dense traffic compared to the proposed CILDOLI-RL model as the proposed CILDOLI-RL is favored by dense traffic conditions in terms of vision.

\subsection{Inference Time}

In autonomous driving; the prediction time of the driving model ultimately determines the rate at which actuators (throttle, brake, steering) can be driven. For high-speed driving; in order to achieve good driving performance, the prediction rate should be high. Therefore, when it comes to a driving model; not only its performance based on navigation capability (CARLA benchmark), collision free driving (NoCRASH benchmark), No other infractions except collisions (NoOTHERINFRACTION) is important; but also the complexity of the model is important for testing at high speeds. We test the driving model's complexity using the average inference time for both CPU and GPU. The tested CPU is a 16 core Intel $11800 \mathrm{H}$ CPU running with Turbo Boost enabled and the GPU is NVidia RTX3060. The results as shown in Table 5 .

Table 5: Complexity Comparison

\begin{tabular}{|l|l|l|l|l|l|}
\hline Resource & $\begin{array}{l}\text { CILRS } \\
{[\mathbf{1 6}]}\end{array}$ & $\begin{array}{l}\text { Modular } \\
{[\mathbf{8}]}\end{array}$ & $\begin{array}{l}\text { CIRL } \\
{[38]}\end{array}$ & $\begin{array}{l}\text { Proposed } \\
\text { CILDO }\end{array}$ & $\begin{array}{l}\text { Proposed } \\
\text { CILDOLI- } \\
\text { RL }\end{array}$ \\
\hline CPU & 31.57 & $\mathbf{1 7 . 6 4}$ & 33.07 & 30.92 & 31.14 \\
\hline GPU & 17.64 & $\mathbf{1 6 . 8 6}$ & 20.85 & 25.00 & 26.14 \\
\hline
\end{tabular}

The modular pipeline approach for autonomous driving has the least average inference time as evident from the results obtained in Table 5. Therefore, if the accuracy can be traded off for higher frame rate at high speed; modular pipeline will be the best model since it has the least computationally complexity for both CPU and GPU inference. On the other hand, proposed models are comparatively computationally less efficient and are more suitable for driving in dense urban environments with a moderate speed. This complexity occurs because of the additional decoder architecture for semantic segmentation, depth detection and in proposed CILDOLI-RL model having additional traffic light state prediction branch.

\section{Conclusion}

This paper proposed two driving models for full Autonomous driving. Results prove that both models are highly suitable for Urban or rural dense driving scenarios since proposed CILDO model and CILDOLI-RL has the highest score and a moderate score for CARLA benchmark respectively. With respect to safety benchmarks, proposed CILDO and proposed CILDOLI-RL model has a moderate score and the highest score for NoCRASH benchmark and a low score and the highest score for novel NoOTHERINFRACTION benchmark introduced in this research respectively in the Urban or rural dense driving environments. Therefore, in dense traffic driving scenarios since both navigation performance and safety are crucial factors, it can be concluded that CILDOLI-RL as the best model out of the two proposed models. These findings will be valuable to optimize autonomous driving in densely populated cities and highways.

\section{Limitations and future work}

Both of the proposed models are relatively computationally complex and perform poor under empty town driving conditions in the rural town. The proposed models do 
not take extended driving functions such as Reverse and Forward Parking Functions at the end and beginning of navigation, lane changing and overtaking into consideration. They are remaining to be addressed as a future work. Further, the performance of the driving models with additional sensors such as LIDAR remains to be addressed.

\section{Other information}

\subsection{Support}

This research did not receive any financial support. This was conducted with the expenses of the sole author.

\section{2. author contributions}

conceptualization - P.A.D.S.N. Wijesekara; methodology - P.A.D.S.N.W.; software, P.A.D.S.N.W.; validation P.A.D.S.N.W. - formal analysis - P.A.D.S.N.W.; investigation - P.A.D.S.N.W.; resources - P.A.D.S.N.W.; data curation - P.A.D.S.N.W.; writing-original draft preparation - P.A.D.S.N.W. ; writing-review and editing - P.A.D.S.N. Wijesekara; visualization - P.A.D.S.N.W.; supervision None

\subsection{Competing interests}

No conflict of interest.

\subsection{Availability of data, code, and other materials}

Data, code will be available after publication from the author.

\section{Author Biography}

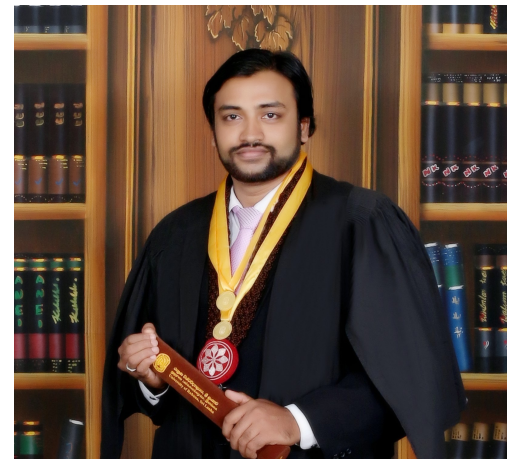

Mr. Shehan Nilmantha Wijesekara is a Lecturer attached to the Department of Electrical and Information Engineering, Faculty of Engineering, University of Ruhuna since 2018. He received his B.Sc. Eng. (First-class Hons.) Degree from Faculty of Engineering, University of Ruhuna, Sri Lanka in 2017. He has been awarded with Mallika De Mel memorial gold medal and Dr. A.D.V. Premaratna memorial gold medal for his Bachelor's degree from the university of Ruhuna. He won best student award for excelling in Electronic Engineering. He has also been awarded Migara Ranatunga award for industrial training and E.W. Karunaratna award for the undergraduate project from IESL. He has also received Chinese ambassador scholarship for academic excellence in 2017. His research interests are in the fields such as Machine learning, AI, Image processing, Statistics, Channel coding, Wireless communication, Data Fusion, Biomedical Engineering.

\section{References}

[1] N. Shalal, T. Low, C. McCarthy and N. Hancock, "A review of autonomous navigation systems in agricultural environments," University of Southern Queensland, 2013

[2] M. Toda, O. Kitani, T. Okamoto and T. Torii, "Navigation method for a mobile robot via sonar-based crop row mapping and fuzzy logic control," Elsevier Journal of Agricultural Engineering Research, vol.72(4), pp.299-309, 1999

[3] E.R. Benson, T.S Stombaugh, N.Noguchi, J.D. Will and J.F. Reid, "An evaluation of a geomagnetic direction sensor for vehicle guidance in precision agriculture applications," Citeseer ASAE paper, vol. 983203, 1998.

[4] R. Gottschalk, X.P. Burgos-Artizzu, A. Ribeiro, G. Pajares and A.S. Miralles, "Real-time image processing for the guidance of a small agricultural field inspection vehicle," International journal of intelligent systems technologies and applications, vol.8(14), pp.434-443, 2010

[5] P.M. Blok, K. van Boheemen, F.K. van Evert, J. IJsselmuiden and G.H. Kim, "Robot navigation in orchards with localization based on Particle filter and Kalman filter," Computers and Electronics in Agriculture, vol. 157, pp.261-269, 2019

[6] J. Libby and G. Kantor, 'Deployment of a point and line feature localization system for an outdoor agriculture vehicle," in 2011 IEEE International Conference on Robotics and Automation, May 2011, pp. $1565-1570$

[7] R. Siegwart, I.R. Nourbakhsh and D. Scaramuzza, Introduction to autonomous mobile robots, Cambridge, MA: MIT press, 2011

[8] Z. Li, , T. Motoyoshi, , K. Sasaki,, T. Ogata, and S. Sugano, "Rethinking self-driving: Multi-task knowledge for better generalization and accident explanation ability", arXiv preprint arXiv:1809.11100, 2018

[9] S. Ross, G. Gordon and D. Bagnell, "A reduction of imitation learning and structured prediction to noregret online learning", in Proceedings of the fourteenth international conference on artificial intelligence and statistics, JMLR Workshop and Conference Proceedings, June 2011, pp. 627-635

[10] L. Sun, C. Peng, W. Zhan and M. Tomizuka, "A fast integrated planning and control framework for autonomous driving via imitation learning", in $D y$ namic Systems and Control Conference, Vol. 51913, pp.V003T37A012, American Society of Mechanical Engineers, September 2018. 
[11] J.Chen, B. Yuan and M. Tomizuka, "Deep imitation learning for autonomous driving in generic urban scenarios with enhanced safety", In 2019 IEEE/RSJ International Conference on Intelligent Robots and Systems (IROS), IEEE, November 2019, pp. 28842890.

[12] D. Pomerleau, "An autonomous land vehicle in a neural network", Advances in Neural Information Processing Systems, Burlington, MA, USA:Morgan Kaufmann Publishers Inc., 1998

[13] H. Xu, Y. Gao, F. Yu and T. Darrell, "End-to-end learning of driving models from large-scale video datasets," In Proceedings of the IEEE conference on computer vision and pattern recognition, 2017, pp. 2174-2182

[14] F. Codevilla, M. Müller, A. López, V. Koltun and A. Dosovitskiy, "End-to-end driving via conditional imitation learning," In 2018 IEEE International Conference on Robotics and Automation (ICRA), IEEE, May 2018, pp. 4693-4700

[15] J. Hawke, R. Shen, , C. Gurau, S. Sharma, D. Reda, , N. Nikolov, P. Mazur, S. Micklethwaite, , N. Griffiths, A. Shah and A. Kndall, Urban driving with conditional imitation learning. In 2020 IEEE International Conference on Robotics and Automation (ICRA), IEEE, May 2020, pp. 251-257

[16] F. Codevilla, E. Santana, A.M. López and A. Gaidon, "Exploring the limitations of behavior cloning for autonomous driving," In Proceedings of the IEEE/CVF International Conference on Computer Vision, 2019, pp. 9329-9338

[17] Q. Zou, H. Jiang, Q. Dai, Y. Yue, L. Chen and Q. Wang, "Robust lane detection from continuous driving scenes using deep neural networks," IEEE transactions on vehicular technology, vol. 69(1), pp.4154,2019

[18] B. Wu, F. Iandola, P.H. Jin and K. Keutzer, "Squeezedet: Unified, small, low power fully convolutional neural networks for real-time object detection for autonomous driving", In Proceedings of the IEEE conference on computer vision and pattern recognition workshops, IEEE, 2017, pp. 129-137

[19] A. Dosovitskiy, G. Ros, F. Codevilla, , A. Lopez, and V. Koltun, "CARLA: An open urban driving simulator," In Conference on robot learning, PMLR, October 2017 , pp. 1-16

[20] P. Henderson, R. Islam, P. Bachman, J. Pineau, D. Precup and D. Meger, "Deep reinforcement learning that matters," In Proceedings of the AAAI conference on artificial intelligence, Vol. 32(1), April 2018

[21] J. Michels, A.Saxena and A.Y. Ng, "High speed obstacle avoidance using monocular vision and reinforcement learning," In Proceedings of the 22nd international conference on Machine learning, August 2005, pp. 593-600
[22] S.Y. Oh, J.H. Lee and D.H. Choi, "A new reinforcement learning vehicle control architecture for visionbased road following," IEEE Transactions on Vehicular Technology, vol. 49(3), pp.997-1005, 2000

[23] C. Desjardins and B. Chaib-Draa, "Cooperative adaptive cruise control: A reinforcement learning approach", IEEE Transactions on intelligent transportation systems, vol.12(4), pp. 1248-1260, 2011

[24] S. Lange, T. Gabel and M. Riedmiller, "Batch reinforcement learning", In Reinforcement learning, Berlin, Heidelberg: Springer, 2012, pp. 45-73

[25] X. Li, X. Xu and L. Zuo, ”Reinforcement learning based overtaking decision-making for highway autonomous driving," In 2015 Sixth International Conference on Intelligent Control and Information Processing (ICICIP), IEEE, November 2015, pp. 336342

[26] S. Kardell and M. Kuosku, "Autonomous vehicle control via deep reinforcement learning", Master's thesis, Chalmers University of Technology, Gothenburg, Sweden, 2017

[27] D. Isele, R. Rahimi, A. Cosgun, K. Subramanian and K. Fujimura, "Navigating occluded intersections with autonomous vehicles using deep reinforcement learning," In 2018 IEEE International Conference on Robotics and Automation (ICRA), IEEE, May 2018, pp. 2034-2039

[28] M. Cutler, T.J. Walsh and J.P. How, "Reinforcement learning with multi-fidelity simulators", In 2014 IEEE International Conference on Robotics and Automation (ICRA), IEEE, May 2014, pp. 3888-3895

[29] C. Ye, H. Ma, X. Zhang, K. Zhang and S. You, "Survival-oriented reinforcement learning model: An efficient and robust deep reinforcement learning algorithm for autonomous driving problem", In International Conference on Image and Graphics, , Springer, September 2017, pp. 417-429

[30] C. Yu, X. Wang, X. Xu, M. Zhang, H. Ge, J. Ren, L. Sun, B.Chen and G.Tan, "Distributed multiagent coordinated learning for autonomous driving in highways based on dynamic coordination graphs," IEEE Transactions on Intelligent Transportation Systems, vol. 21(2), pp.735-748, 2019

[31] S. Wang, D. Jia and X. Weng, "Deep reinforcement learning for autonomous driving", arXiv preprint arXiv:1811.11329, 2018

[32] P.Wang, C.Y. Chan and A. de La Fortelle, "A reinforcement learning based approach for automated lane change maneuvers," In 2018 IEEE Intelligent Vehicles Symposium (IV), IEEE, June 2018, pp. 1379-1384

[33] J. Chen, B. Yuan and M. Tomizuka, "Model-free deep reinforcement learning for urban autonomous driving," In 2019 IEEE intelligent transportation 
systems conference (ITSC), IEEE, October 2019, pp. 2765-2771

[34] A.E. Sallab, M. Abdou, E. Perot and S. Yogamani, "Deep reinforcement learning framework for autonomous driving," Electronic Imaging, Society for Imaging Science and Technology, vol. 2017(19), pp.70-76, 2017

[35] F.M. Favarò , N. Nader, S.O. Eurich , M. Tripp and N. Varadaraju, "Examining accident reports involving autonomous vehicles in California," PLOS ONE, vol. 12(9), pp. e0184952, 2017

[36] D. Wakabayashi, "Self-Driving Uber Car Kills Pedestrian in Arizona, Where Robots Roam," nytimes.com, Mar.19, 2018.[Online]Available: https://www . nytimes.com/2018/03/19/ technology/uber-driverless-fatality . html.[Accessed: Sept.16, 2021].

[37] A. Sauer, N.Savinov and A.Geiger, "Conditional affordance learning for driving in urban environments," In Conference on Robot Learning ,PMLR, Oct. 2018, pp. 237-252

[38] X. Liang, T.Wang, L. Yang and E. Xing, ”CIRL: Controllable imitative reinforcement learning for vision-based self-driving," In Proceedings of the European Conference on Computer Vision (ECCV), 2018 , pp. 584-599

[39] M. Müller, V. Casser, J.Lahoud, , N. Smith and B. Ghanem, "Sim4cv: A photo-realistic simulator for computer vision applications,", International Journal of Computer Vision, vol. 126(9), pp. 902-919, 2018

[40] Carla Simulator, "3rd. Maps and navigation", carla.readthedocs.io, 2021.[Online]Available: https: //carla.readthedocs.io/en/latest/ core_map/.[Accessed: Sept.20, 2021]

[41] F. Codevilla, A.M. Lopez, V. Koltun and A. Dosovitskiy, "On offline evaluation of vision-based driving models," In Proceedings of the European Conference on Computer Vision (ECCV), 2018, pp. 236251
[42] N. Srivastava, G. Hinton, A. Krizhevsky, I. Sutskever and R. Salakhutdinov, "Dropout: a simple way to prevent neural networks from overfitting," The journal of machine learning research, vol. 15(1), pp.1929-1958, 2014

[43] Keras API reference, "Keras Applications", keras.io, 2021.[Online]Available: https: //keras.io/api/applications/.[Accessed: Sept.21, 2021]

[44] O. Ronneberger, P. Fischer and T. Brox, "U-net: Convolutional networks for biomedical image segmentation", In International Conference on Medical image computing and computer-assisted intervention, Springer, Oct. 2015, pp. 234-241

[45] M. Sandler, A. Howard, M. Zhu, A. Zhmoginov and L.C. Chen, "Mobilenetv2: Inverted residuals and linear bottlenecks," In Proceedings of the IEEE conference on computer vision and pattern recognition, 2018, pp. 4510-4520

[46] P. Isola, J.Y. Zhu, T. Zhou and A.A. Efros, "Imageto-image translation with conditional adversarial networks," In Proceedings of the IEEE conference on computer vision and pattern recognition, 2017, pp. 1125-1134

[47] Google LLC, "Google Maps",google.com, 2021.[Online].Available: https://www . google. com/maps/.[Accessed: Oct.23, 2021]

[48] M. Van Otterlo and M.Wiering, "Reinforcement learning and markov decision processes", In Reinforcement learning, Berlin, Heidelberg: Springer, 2012, pp. 3-42

[49] C.J. Watkins and P. Dayan, "Q-learning," Machine learning, vol. 8(3-4), pp.279-292, 1992

[50] T.P. Lillicrap, J.J. Hunt, A. Pritzel, N. Heess, T. Erez, Y. Tassa, D. Silver and D. Wierstra, "Continuous control with deep reinforcement learning," arXiv preprint arXiv:1509.02971, 2015.

[51] Carla Simulator, "Cameras and Sensors," carla.readthedocs.io, [Online].Available: https://carla.readthedocs.io/en/stable/ cameras_and_sensors/.[Accessed: Sep.8, 2021] 